\title{
Primary Axillary Lipogranuloma in a Patient With Systemic Lupus Erythematosus
}

\author{
Suk Jung Kim \\ Department of Radiology, Haeundae Paik Hospital, Inje University College of Medicine, Busan, South Korea
}

Lipogranuloma is an uncommon inflammatory reactive process related to endogenous or exogenous lipid materials. Although lipogranuloma most frequently involves the penis and scrotum of young adults, it may affect many other organs. This report describes primary axillary lipogranuloma in a 45-year-old woman with systemic lupus erythematosus in the light of the mammographic and ultrasonographic findings of this rare entity. This is the first report of primary axillary lipogranuloma in the literature in English.

Keywords: Axilla; lipogranuloma; systemic lupus erythematosus.

Lipogranuloma or sclerosing lipogranuloma is an unusual benign granulomatous reaction to exogenous or endogenous lipids. ${ }^{1,2}$ Although this benign process may involve various organs, there has only been one report of axillary localization in the literature in English, which was secondary to silicone implant rupture and did not include underlying systemic disease. ${ }^{2}$

To the best of our knowledge, this report describes the first case of axillary lipogranuloma in a patient with systemic lupus erythematosus (SLE) in the light of mammographic and ultrasonographic findings of this rare entity.

\section{CASE REPORT}

A 45-year-old woman was admitted to our hospital for the evaluation of a painless and palpable lump in her right axilla. The patient discovered the mass two weeks ago. The mass was poorly defined and felt soft upon palpation. The patient history had no trauma injection of exogenous substances or topical application of oily substances in the affected area.

Full-field digital mammography was performed (MAMMOMAT Inspiration; Siemens AG, Munich, Germany). A right mediolateral oblique view revealed an asymmetric density in the right axilla (Figure 1). Ultrasonography was performed with a 5- to $12-\mathrm{MHz}$ linear transducer (iU22; Philips Medical Systems, Bothell, WA, USA), which identified a poorly defined, elongated, homogeneously hyperechoic lesion in the subcutaneous fat layer of the right axilla (Figure 2). The lesion did not exhibit increased vascularity, except for a normal traversing venous structure (Figure 2). The lesion was surgically excised. Pathological examination confirmed the diagnosis as lipogranuloma (Figure 3). On the other hand, complete blood test results were normal. 


\section{DISCUSSION}

Lipogranuloma or sclerosing lipogranuloma is known to be a tissue reaction to exogenous foreign bodies or endogenous lipids, and its etiology has not yet been clearly defined. ${ }^{3,4}$ This disease has been classified into two types according to its causative factors. ${ }^{3}$ The primary type is caused by the breakdown of endogenous lipids, and the secondary type is caused by injection of exogenous foreign materials such as paraffin, mineral or vegetable oils, and silicone. ${ }^{5}$

Furthermore, primary systemic sclerosing lipogranulomas may be associated with underlying systemic diseases such as lipid metabolism disorders, diabetes mellitus, and nephritic syndrome, which may cause lipid accumulation in tissues. ${ }^{2}$ Allergic mechanisms have also been suspected as an underlying cause of primary sclerosing lipogranuloma. ${ }^{3}$ T-cell mediated immune responses were proposed as the mechanism responsible for the formation of primary lipogranuloma of the scrotum. ${ }^{3}$ In some cases, endogenous lipid degeneration related directly or by allergic mechanisms to heat, cold, and trauma has also been suggested as a

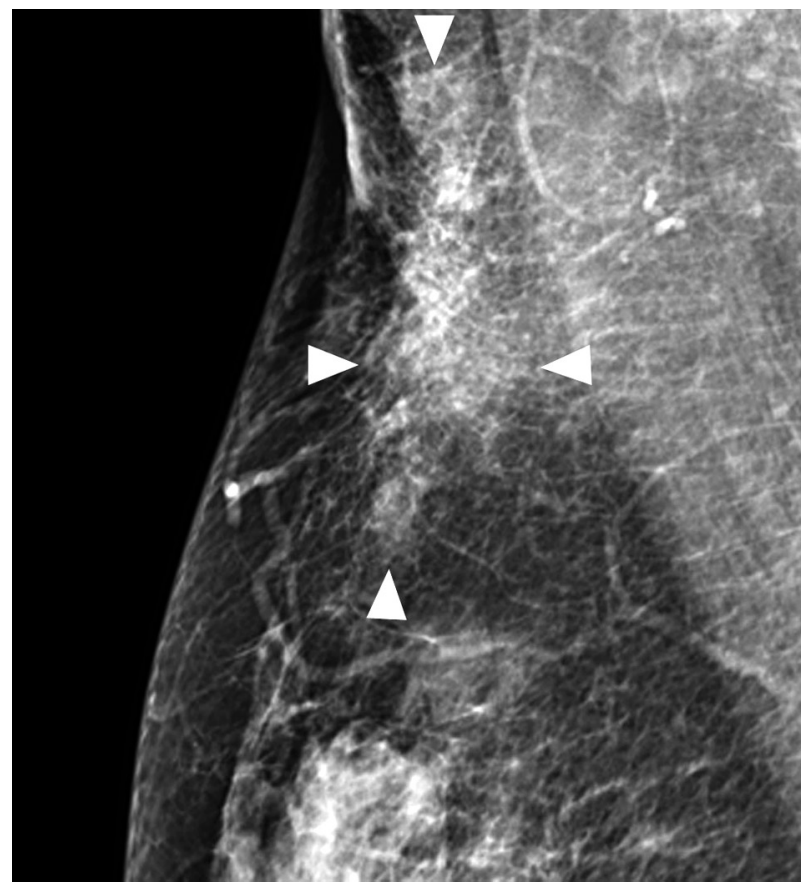

Figure 1. A right mediolateral view upon mammography showing an ill-defined, isodense lesion (arrow heads) interspersed with fat densities in the right axilla. causative factor. ${ }^{4}$ However, other cases of primary lipogranuloma have been reported without any plausible history or evidence of causal factors in the literature. ${ }^{4}$ Secondary lipogranuloma usually develops subcutaneous injection of foreign bodies for cosmetic or reconstructive purposes, or topical use of oily drug preparations..$^{2,4}$
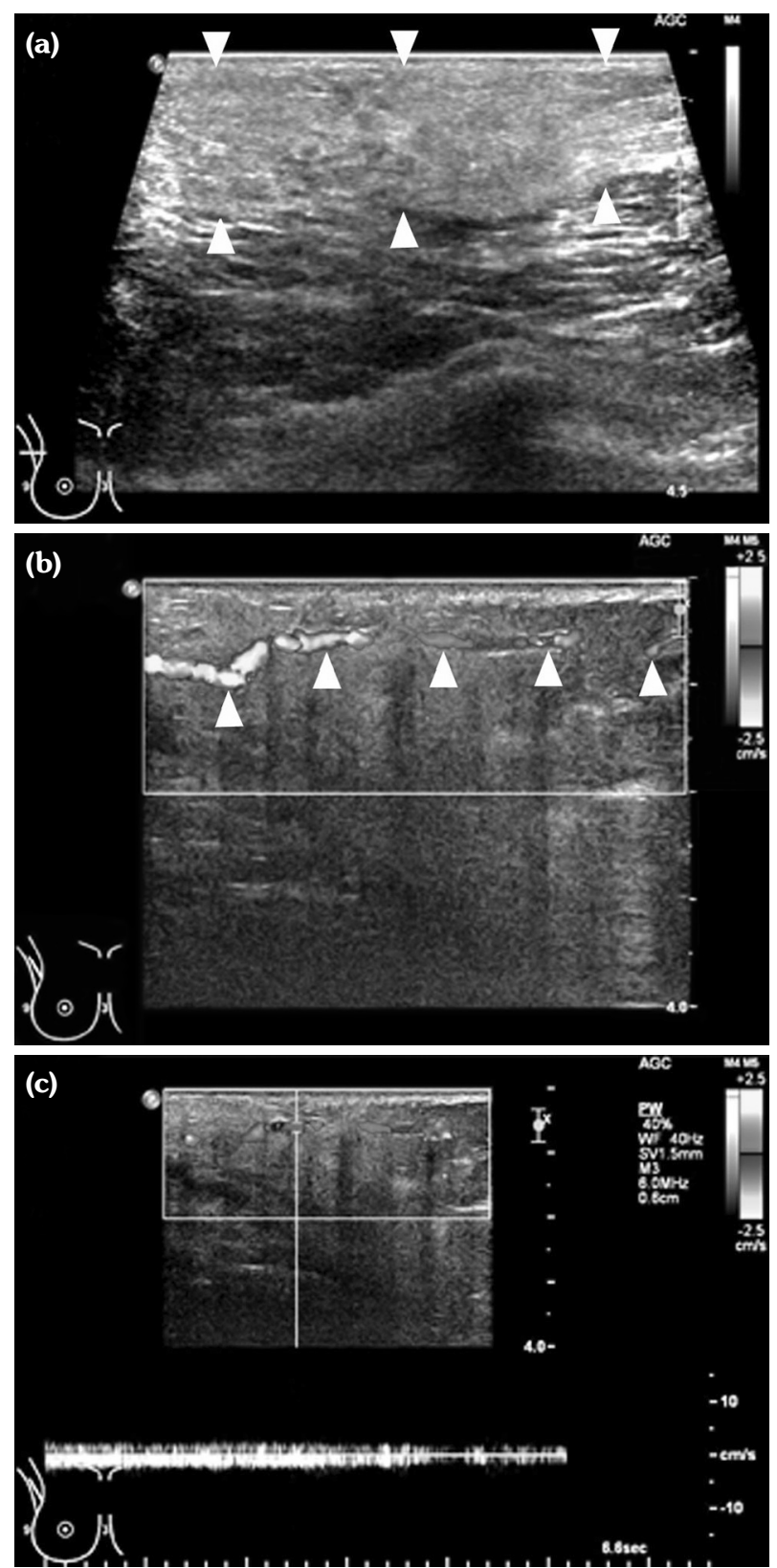

Figure 2. (a) A grayscale ultrasonogram showing an ill-defined, elongated, homogeneously hyperechoic lesion in the subcutaneous fat layer of the right axilla (arrow heads). (b, c) Color Doppler and spectral Doppler ultrasonograms showing only a venous structure traversing the axillary lesion (arrow heads). 


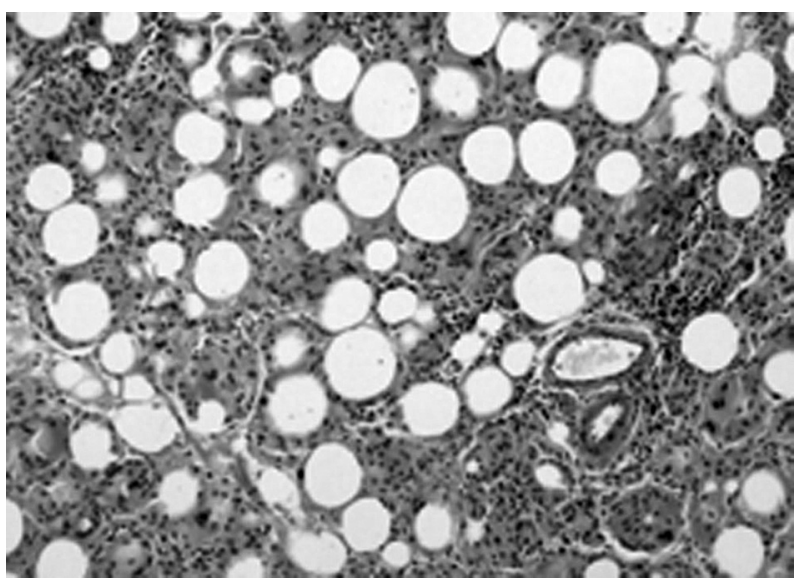

Figure 3. Pathological examination of the excisional biopsy showing a typical lipogranuloma with numerous lipid vacuoles surrounded by multinucleated giant cells, foamy histiocytes, and infiltration of lymphoplasma cells (H-E x 100).

Lipogranulomas mostly occur in the genitourinary system, however, various organs such as the spleen, liver, mesentery, breast, scalp, eyelid, and nose have also been affected. ${ }^{5}$

\section{Histopathologically, lipogranuloma} is characterized by multiple fat vacuoles surrounded by granulomatous tissue composed of multinucleated giant cells, epithelioid histiocytes, and fibrosis. ${ }^{2,6}$ Regardless of the kind of exogenous material responsible for secondary lipogranuloma including paraffin, petrolatum, mineral oils, lanolin, and silicone, a similar histopathological appearance is produced after injection. ${ }^{6}$

Based on all these findings, we assumed that the current case was of primary lipogranuloma of the axilla. Since the metabolic disturbances in SLE include altered lipid profiles, ${ }^{7}$ it seems reasonable that SLE resulted in endogenous fat degeneration in the current case. This patient showed neither eosinophilic infiltrates in the lesion nor eosinophilia in the peripheral blood.

The previous reported case of axillary lipogranuloma differed from the current case in that it resulted from a silicone breast implant rupture, and developed in the axillary lymph nodes. Mammographic and ultrasonographic findings were not available in the previous case.

In the breast, lipogranulomas have been reported after the release of the silicone gel from breast implants, after the application of a traumatic treatment called "Bu-Hwang," and after phenothiazine therapy. 8,9 The mammographic features of lipogranuloma have been described in only two cases. These cases presented a bizarreshaped lesion with bright density which proved to be lipogranuloma with osseous metaplasia and an irregular mass containing several microcalcifications. ${ }^{8,10}$ The ultrasonographic features of breast lipogranuloma have been described in only one case, which presented an ill-defined hyperechoic lesion. ${ }^{10}$

Besides the breast and axilla, the ultrasonographic features of lipogranuloma have been described in the penis and scrotum, and have included cases with an ill-defined area of increased echogenicity in the subcutaneous tissue of the penile shaft, ${ }^{11}$ a poorly defined extratesticular echogenic mass with an elongated appearance on a longitudinal scan, ${ }^{1}$ and an intratesticular heterogeneously hypoechoic mass. ${ }^{12}$

Axillary lesions related to underlying SLE also include reactive lymph adenopathy, lupus panniculitis (profundus), and ectopic axillary breast. $^{13-15}$

In conclusion, we present a case of primary axillary lipogranuloma in a patient with SLE for the first time. To the best of our knowledge, our case presented herein is unique with regards to the affected site, the likelihood of association with systemic disease, and the absence of other precipitating factors. This rare manifestation may broaden the differential clinical diagnosis of axillary lesions in patients with SLE.

\section{Declaration of conflicting interests}

The authors declared no conflicts of interest with respect to the authorship and/or publication of this article.

\section{Funding}

The authors received no financial support for the research and/or authorship of this article.

\section{REFERENCES}

1. Jung SE, Lee JM, Kang CS, Cho YH. Sclerosing lipogranuloma of the scrotum: sonographic findings and pathologic correlation. $\mathrm{J}$ Ultrasound Med 2007;26:1231-3. 
2. Gundeslioglu AO, Hakverdi S, Erdem O, Ozen EC, Inan I, Emlik D. Axillary lipogranuloma mimicking carcinoma metastasis after silicone breast implant rupture: a case report. J Plast Reconstr Aesthet Surg 2013;66:e72-5.

3. Terada T, Minami S, Onda H, Inatsuchi H, Sekido Y, Shimamura K, et al. Primary sclerosing lipogranuloma with broad necrosis of the scrotum. Pathol Int 2003;53:121-5.

4. Akbulut M, Utku Y, Soysal S. Lipogranuloma of the cervix in a postmenopausal patient with a uterine prolapse. Arch Gynecol Obstet 2008;277:277-9.

5. Lim HK, Byun JH, Yoon SE, Kim AY, Jang SJ, Ha HK. Primary sclerosing lipogranuloma of the rectum: CT findings. Br J Radiol 2006;79:e190-2.

6. Kayaselçuk F, Kayaselçuk U, Ozerdem OR, Tuncer I. Posttraumatic lipogranuloma of the hand. Ann Plast Surg 2002;48:223-4.

7. Wu T, Xie C, Han J, Ye Y, Weiel J, Li Q, et al. Metabolic disturbances associated with systemic lupus erythematosus. PLoS One 2012;7:e37210.

8. Lee HH, Park SH, Choi HY, Park HK. Lipogranuloma with osseous metaplasia in the breast that developed after "Bu-Hwang" oriental medicine treatment. Yonsei Med J 2011;52:373-6.

9. Hunter-Craig ID, Tuddenham EG, Earle JH. Lipogranuloma of the breast due to phenothiazine therapy. Br J Surg 1970;57:76-9.

10. Yu M, Bui MM, Gulati P, Masood S. Lipogranuloma. Breast J 2001;7:363-4.

11. Nishizawa K, Kobayashi T, Ogura K, Ide Y, Togashi K. Magnetic resonance imaging of sclerosing lipogranuloma of male genitalia. J Urol 2002;168:1500-1.

12. Ricchiuti VS, Richman MB, Haas CA, Desai D, Cai DX. Sclerosing lipogranuloma of the testis. Urology 2002;60:515.

13. Herman M, Van Ongeval Ch, Van Steen A. Reactive breast and axillary lesions in systemic lupus erythematosus. JBR-BTR 2009;92:154-5.

14. Bacanli A, Uzun S, Ciftcioglu MA, Alpsoy E. A case of lupus erythematosus profundus with unusual manifestations. Lupus 2005;14:403-5.

15. Ben Dhaou B, Boussema F, Aydi Z, Baili L, Rokbani L. Ectopic Axillary Breast during Systemic Lupus. Case Rep Med 2012;2012:403932. 